regarding axial inflammation in spondyloarthritis (SpA), better efficacy regarding cutaneous psoriasis, but is inferior in inflammatory bowel disease (IBD). However, the efficacy of SEC compared to TNFi in anterior uveitis (AU) has not been extensively studied.

Objectives: To compare the occurrence of $A U$ in patients with SpA treated with SEC, adalimumab (ADA), infliximab (IFX) or etanercept (ETN), in clinical practice. Methods: Patients with ankylosing spondylitis or undifferentiated SpA starting either SEC, ADA, IFX or ETN, in 2015 through 2017, were identified in the Swedish Rheumatology Quality register, and were linked to the national patient register for identification of $\mathrm{AU}$. AU-flares were defined as the number of visits with an AU diagnosis, separated by $a \geq 60$ days penalty interval, within ophthalmology outpatient care, during the respective bDMARD treatment.

Follow-up started at the bDMARD initiation, and ended at the first of Dec $31^{\text {st }}$ 2017, death, emigration or discontinuation date of the bDMARD.

To assess and accommodate treatment channeling, crude incidence rates for AU-flares were determined $(A)$ for all bDMARD treatment starts, $(B)$ excluding patients with an $A U$ diagnosis during the year prior to the bDMARD start, and (C) in addition, excluding all first line bDMARD treatment starts.

Hazard ratios (HR) for time until a first on-treatment $A U$ diagnosis were estimated using Cox regression (ADA=reference), adjusted for sex, age, and any history of $A U$, and estimating robust confidence intervals to account for the individuals contributing multiple lines of treatment.

Results: In total, 2,684 patients (52\% women) contributed 3,255 treatment initiations. SEC was less frequently used as first line bDMARD and there was channeling of patients with previous AU, towards treatment with ADA, and away from ETN (Table 1). Further, AU occurred almost exclusively in patients with a pre-treatment history of AU (data not shown).

Table 1.

\begin{tabular}{|c|c|c|c|c|c|c|c|}
\hline Analysis & $\begin{array}{l}\text { Treat-ment } \\
\text { starts, } \mathrm{N}\end{array}$ & $\begin{array}{c}\text { Previous } \\
\text { AU }^{1}\end{array}$ & $\begin{array}{l}\text { Age at treat- } \\
\text { ment start, } \\
\text { mean (sd) }\end{array}$ & $\begin{array}{l}\text { N Previous } \\
\text { bDMARD, } \\
\text { median }\end{array}$ & $\begin{array}{c}\text { HR }(95 \% \\
\text { CI) for first } \\
\text { AU-diagnosis }\end{array}$ & $\begin{array}{c}\mathrm{AU} \\
\text { flares, } \\
\mathrm{N}\end{array}$ & $\begin{array}{c}\text { Follow-up }{ }^{2} \\
\text { years }\end{array}$ \\
\hline \multicolumn{8}{|c|}{ A. All treatment starts, $\mathrm{N}=3255$} \\
\hline SEC & 333 & $21 \%$ & $48(13)$ & 2 & $2.0(1.2-3.3)$ & 52 & 241 \\
\hline ADA & 872 & $34 \%$ & $44(12)$ & 1 & Ref & 175 & 973 \\
\hline IFX & 714 & $21 \%$ & $43(14)$ & 0 & $0.9(0.6-1.4)$ & 68 & 677 \\
\hline ETN & 1336 & $17 \%$ & $44(14)$ & 0 & $0.9(0.7-1.3)$ & 102 & 1290 \\
\hline \multicolumn{8}{|c|}{ B. Excluding patients with prior AU within 1 year before treatment start, $\mathrm{N}=2907$} \\
\hline SEC & 304 & $13 \%$ & $47(13)$ & 2 & $3.1(1.4-7.3)$ & 10 & 212 \\
\hline ADA & 711 & $19 \%$ & $44(13)$ & 1 & Ref & 18 & 792 \\
\hline IFX & 633 & $11 \%$ & $43(14)$ & 0 & $1.0(0.4-2.3)$ & 8 & 599 \\
\hline ETN & 1259 & $12 \%$ & $44(14)$ & 0 & $1.8(1.0-3.4)$ & 43 & 1204 \\
\hline \multicolumn{8}{|c|}{ C. Excluding patients with prior AU within 1 year before treatment start and first line } \\
\hline \multicolumn{8}{|c|}{ bDMARD, N=1288 } \\
\hline SEC & 284 & $14 \%$ & $48(13)$ & 2 & $2.5(1.0-6.2)$ & 10 & 198 \\
\hline ADA & 374 & $18 \%$ & $45(13)$ & 1 & Ref & 11 & 384 \\
\hline IFX & 185 & $12 \%$ & $45(14)$ & 2 & $1.3(0.4-4.0)$ & 4 & 166 \\
\hline ETN & 445 & $17 \%$ & 47 (14) & 1 & $1.9(0.9-4.0)$ & 23 & 439 \\
\hline
\end{tabular}

1) Anterior uveitis between 2001 and treatment start; 2) Total follow-up time for analyses of incidence rate.

The incidence rates of AU-flares were higher for SEC and ETN compared to ADA and IFX, in the analyses $(B, C)$ accommodating for channeling, figure 1 .

Compared to ADA, the adjusted HRs of a first on-treatment AU-diagnosis were also higher for SEC and ETA, Table 1.
Conclusion: In clinical practice, SEC and ETN are associated with a higher incidence of AU than ADA and INF, suggesting a poorer protective effect of SEC and ETN against AU. These preliminary results should be interpreted in light of pronounced treatment channeling, which was only partly accommodated for.

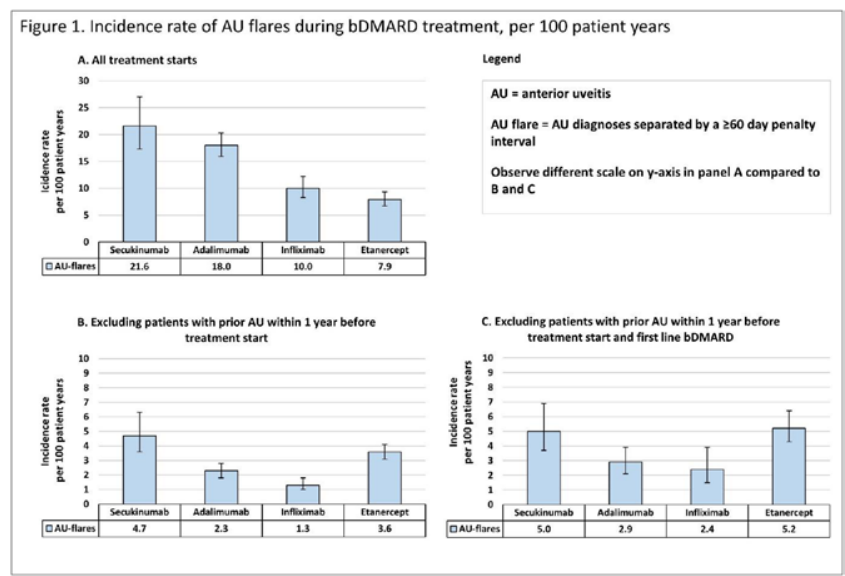

Disclosure of Interests: Ulf Lindström: None declared, Karin Bengtsson: None declared, Tor Olofsson: None declared, Daniela Di Giuseppe: None declared, Bente Glintborg Grant/research support from: Grants from Pfizer, Biogen and Abbvie, Helena Forsblad-d'Elia Grant/research support from: Unrestricted grant from Novartis., Consultant of: Advisory Board Fees from Sandoz, Novartis, and Abbvie, Lennart T.H. Jacobsson Consultant of: AbbVie, Eli Lilly, Janssen, Novartis and Pfizer, Johan Askling Grant/research support from: JA acts or has acted as PI for agreements between Karolinska Institutet and the following entities, mainly in the context of the ARTIS national safety monitoring programme of immunomodulators in rheumatology: Abbvie, BMS, Eli Lilly, Merck, MSD, Pfizer, Roche, Samsung Bioepis, Sanofi, and UCB Pharma

DOI: 10.1136/annrheumdis-2020-eular.234

\section{OP0015 \\ SEX-SPECIFIC U-SHAPED RELATIONSHIP BETWEEN SERUM URATE AND MORTALITY IN THE UNITED STATES GENERAL POPULATION}

K. D'silva ${ }^{1}$, C. Yokose ${ }^{1}$, L. Lu², Y. Zhang ${ }^{1}$, H. Choi ${ }^{1} .{ }^{1}$ Massachusetts General Hospital, Division of Rheumatology, Allergy, and Immunology, Boston, United States of America; ${ }^{2}$ Arthritis Research Canada, Richmond, Canada

Background: A U-shaped relationship may exist between serum urate (SU) and cardiovascular events, although the relationship between SU and mortality is unclear. The most recent EULAR recommendations for gout advise agains maintaining $\mathrm{SU}<3 \mathrm{mg} / \mathrm{dl}$ for prolonged periods.

Objectives: To examine the relationship between SU and all-cause and cause-specific mortality in large cohorts in the United States (US).

Methods: We examined National Health and Nutrition Examination Survey (NHANES) data from 1988-1994 (NHANES III) and 1999-2007 including subjects

Table. Multivariable hazard ratios for all-cause and cause-specific mortality in NHANES III and 1999-2007.

\begin{tabular}{|c|c|c|c|c|c|c|}
\hline \multicolumn{7}{|c|}{ Men } \\
\hline Serum urate range $(\mathrm{mg} / \mathrm{dL})$ & $<4$ & 4-5 & $5-6$ & $6-7$ & $7-8$ & $>8$ \\
\hline Deaths $(\mathrm{N})$ & 325 & 934 & 1513 & 1412 & 872 & 658 \\
\hline All-cause & $1.33(1.17,1.51)$ & $1.17(1.07,1.28)$ & $1.00^{\star *}$ & $1.04(0.96,1.13)$ & $1.13(1.04,1.24)$ & $1.52(1.37,1.69)$ \\
\hline Cardiovascular & $0.97(0.75,1.27)$ & $1.10(0.93,1.30)$ & 1.00 & $0.93(0.80,1.08)$ & $1.13(0.96,1.34)$ & $1.22(1.00,1.49)$ \\
\hline $\begin{array}{l}\text { Chronic lower respiratory } \\
\text { disease }\end{array}$ & $0.92(0.51,1.68)$ & $0.96(0.64,1.43)$ & 1.00 & $1.01(0.69,1.47)$ & $0.86(0.53,1.38)$ & $1.91(1.18,3.10)$ \\
\hline Alzheimer's disease & $1.13(0.50,2.55)$ & $0.88(0.48,1.61)$ & 1.00 & $1.14(0.69,1.91)$ & $0.87(0.45,1.69)$ & $0.23(0.05,0.99)$ \\
\hline Diabetes mellitus & $3.39(1.89,6.09)$ & $1.81(1.10,3.00)$ & 1.00 & $0.86(0.51,1.44)$ & $0.93(0.51,1.69)$ & $1.60(0.87,2.95)$ \\
\hline \multicolumn{7}{|c|}{ Women } \\
\hline Serum urate range $(\mathrm{mg} / \mathrm{dL})$ & $<3$ & 3-4 & 4-5 & $5-6$ & $6-7$ & $>7$ \\
\hline Deaths (N) & 181 & 750 & 1321 & 1170 & 776 & 703 \\
\hline All-cause & $1.11(0.93,1.31)$ & $1.03(0.93,1.14)$ & $1.00(0.92,1.09)$ & 1.00 & $1.11(1.00,1.22)$ & $1.45(1.31,1.61)$ \\
\hline Cardiovascular & $1.23(0.88,1.71)$ & $1.11(0.92,1.34)$ & $0.94(0.80,1.11)$ & 1.00 & $1.06(0.89,1.28)$ & $1.16(0.95,1.41)$ \\
\hline $\begin{array}{l}\text { Chronic lower respiratory } \\
\text { disease }\end{array}$ & $1.26(0.62,2.57)$ & $1.11(0.72,1.72)$ & $0.87(0.58,1.30)$ & 1.00 & $0.66(0.39,1.12)$ & $0.85(0.49,1.45)$ \\
\hline Alzheimer's disease & $1.38(0.57,3.36)$ & $0.97(0.54,1.73)$ & $1.15(0.73,1.81)$ & 1.00 & $0.79(0.44,1.41)$ & $0.54(0.25,1.15)$ \\
\hline Diabetes mellitus & $1.69(0.71,4.02)$ & $1.50(0.89,2.53)$ & $0.91(0.56,1.46)$ & 1.00 & $1.11(0.68,1.82)$ & $1.66(1.02,2.71)$ \\
\hline
\end{tabular}


aged $\geq 18$ with an enrollment SU measurement. We used Cox proportional hazards regression models to estimate sex-specific mortality risk relative to a referent SU 5-6 mg/dL, adjusting for NHANES cycle, age, race, body mass index (BMI), education, alcohol use, smoking, hypertension, total cholesterol, estimated glomerular filtration rate (GFR), and competing risks, using age as a time scale for survival analysis. Results: Among 19,954 men and 21,853 women, there were 5,714 male deaths and 4,901 female deaths (median follow-up $14.2 \pm 6.9$ years). Among men, there was a $33 \%$ increased all-cause mortality risk at SU $<4 \mathrm{mg} / \mathrm{dL}$ ( $\mathrm{HR} 1.33,95 \% \mathrm{Cl} 1.17-$ $1.51)$ and $52 \%$ increased all-cause mortality risk at SU $>8 \mathrm{mg} / \mathrm{dL}$ (HR $1.52,95 \% \mathrm{Cl}$ 1.37-1.69) compared to subjects with SU $5-6 \mathrm{mg} / \mathrm{dL}$, driven by cause-specific mortality from diabetes at low SU and chronic lower respiratory diseases and cardiovascular disease at high SU (Table). In women, there was no increased mortality risk at low SU and a $45 \%$ increased all-cause mortality risk at SU $>7 \mathrm{mg} / \mathrm{dL}$ ( $\mathrm{HR} 1.45, \mathrm{Cl}$ 1.31-1.61) compared to subjects with SU $5-6 \mathrm{mg} / \mathrm{dL}$, driven by cause-specific mortality from diabetes. Mortality from Alzheimer's disease was lower at high SU among men (HR 0.23, 95\% Cl 0.05-0.99) and women (HR 0.54, 95\% Cl 0.25-1.15).

Conclusion: In large cohorts representative of the US population, there was a U-shaped relationship between SU and all-cause mortality in men but not women. In men with low SU, mortality was driven primarily by diabetes, which may be explained by the uricosuric effect of uncontrolled hyperglycemia in diabetes patients. The lower mortality from Alzheimer's disease at high SU agrees with previously shown inverse associations between gout and Alzheimer's disease. Further studies are needed to determine the presence of causality underlying these associations. Disclosure of Interests: Kristin D'Silva: None declared, Chio Yokose: None declared, Leo Lu: None declared, Yuqing Zhang: None declared, Hyon Choi Grant/research support from: Ironwood, Horizon, Consultant of: Takeda, Selecta, Horizon, Kowa, Vaxart, Ironwood

DOI: 10.1136/annrheumdis-2020-eular.2229

\section{OP0016 GUT MICROBIOTA DYSBIOSIS IN THE HIGH-RISK INDIVIDUAL FOR RA TRIGGERS THE MUCOSAL IMMUNITY PERTURBATION AND PROMOTES RHEUMATOID ARTHRITIS DEVELOPMENT}

Y. Tong ${ }^{1}$, Y. Zhao ${ }^{1}$, Y. Liu' ${ }^{1}$, Y. Luo ${ }^{1} .{ }^{1}$ West China Hospital, Sichuan University, Department of Rheumatology and Immunology, Chengdu, China

Background: The early treatment of rheumatoid arthritis (RA) is associated with better outcomes. In recent years, studies in our understanding of the preclinical events in RA help to define the "at-risk" populations who might go on to develop RA. Emerging evidence indicate that initiating events may occur at mucosal surfaces including oral cavity, lung and gut influenced by the local microbiome. Therefore, identifying the microbiome characteristics in prospective cohorts of at-risk individuals enables risk prediction or prevention of RA.

Objectives: Here, we undertook this study to clarify the intestinal microbiota changes in individuals at high risk for RA. Meanwhile, we performed fecal transplantation study to investigate whereby the intestinal dysbiosis in the pre-RA population contributes to RA initiation and development, and provide a new prevention strategy for the treatment of this disease.

Methods: 42 high-risk for RA individuals (Pre-RA), who were defined as having a positive serum antibody for anti-cyclic citrullinated peptide (CCP), 31 RA patients and 38 healthy individuals $(\mathrm{HC})$ were recruited in this study. The V3-V4 region of $16 \mathrm{~S}$ ribosomal RNA of fecal samples from these individuals were sequenced. We evaluated the gut permeability and the gut barrier dysfuction using HE staining and RT-PCR in mice receiving fecal transplantation (FMT). Flow cytometry was applied to measure the proportions of T cell subsets in immune organs. The disease severity of collagen-induced arthritis $(\mathrm{ClA})$ was also evaluated after the mice receiving FMT.

Results: Alpha diversity analysis showed a comparable community richness and a lower community diversity of the intestinal microbiota in Pre-RA compared to $\mathrm{HC}($ Fig $1 \mathrm{~A})$. At the family level, the abundance of Bacteroidaceae gradually decreased from $\mathrm{HC}$ to Pre-RA individuals and to RA patients (Fig 1B). On the contrary, the enriched abundances of Streptococcaceae, Lactobacillus, Lactococcus, Weissella and unclassified_o_Lactobacillales were observed in RA patients (Fig 1B). There was different intestinal microbiota construction between groups based on principal coordinate analysis (PCoA). The intestinal microbiota communities dynamically shifted from $\mathrm{HC}$ to Pre-RA and to RA patients (Fig.1C). Fecal transplantation study showed that gut microbiota from Pre-RA group $(P)$ significantly increased the fluorescence intensity (Fig 2A), accompanied with a significantly decreased ZO-1 gene expression (Fig 2B), and injured epithelial microvilli of the small intestine (Fig 2C). Moreover, the percentages of Th17 cells in the mesenteric lymph nodes $(\mathrm{mLN})$ and peyer patches (PP) were also significantly increased in P and $\mathrm{R}$ groups (Fig 2D, E). Importantly, in CIA models, the joints redness and swelling in the mice receiving Pre-RA faeces occurred earlier and were more severe compared to $\mathrm{HC}$-transplanted mice (Fig $2 \mathrm{~F}, \mathrm{G}$ and $\mathrm{H}$ ).

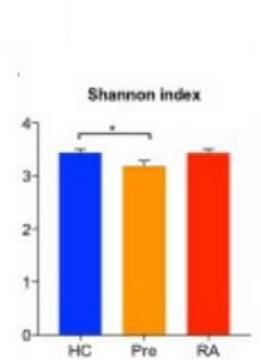

C

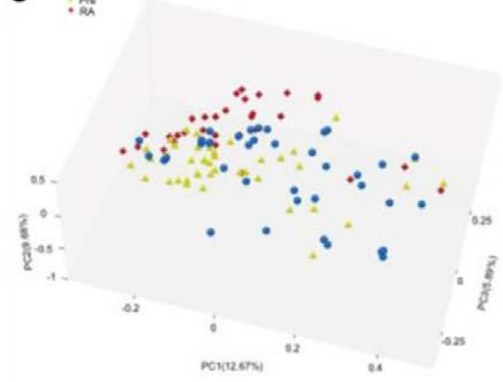

B

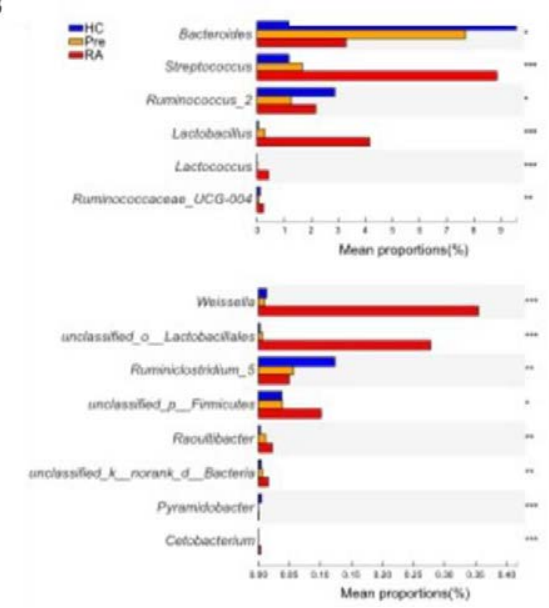

Figure 1.

A

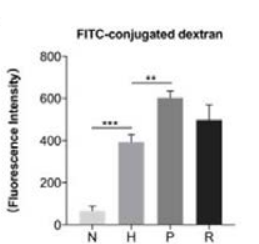

B

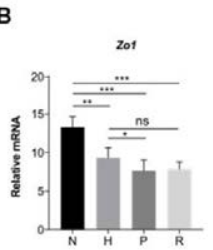

C

D
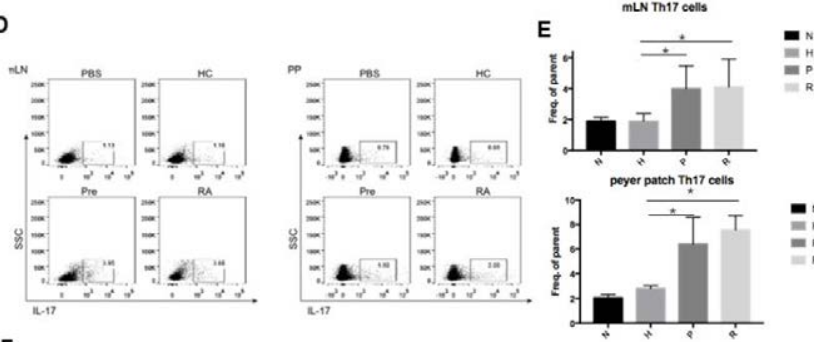

$\mathbf{F}$

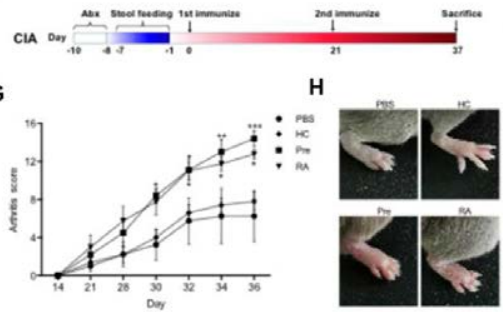

Figure 2.

Conclusion: The intestinal microbiota changed gradually during disease progression of human rheumatoid arthritis. The gut microbiota from Pre-RA individuals can trigger the gut barrier dysfunction and intestinal mucosal immunity imbalance, which may further contribute to the arthritis initiation and development. References:

[1] Brusca, S. B., Abramson, S. B. \& Scher, J. U. Microbiome and mucosal inflammation as extra-articular triggers for rheumatoid arthritis and autoimmunity. Curr Opin Rheumatol 26, 101-107, doi:10.1097/bor.0000000000000008 (2014). 